\title{
Interview
}

\section{Metadata explained: The evolution of schemas, taxonomies and ontologies in today's DAM systems - An Interview with Tom Bachmann of EAS Planning}

\section{Tom Bachmann}

specializes in metadata/taxonomies for digital asset management. He is a principal at EAS Planning, a consultancy that he co-founded in 2001. Tom combines expertise in library science with a background in strategic planning and user experience research. His work includes developing DAM solutions for a range of media firms, for digital library collections and for technology vendors. Tom holds a BA from Wesleyan University and a Masters in Library \& Information Science from Florida State University with a concentration in Information Architecture.

ABSTRACT In this interview, Tom and Michael explore the current state of metadata structures - ranging from taxonomies to ontologies - in DAM implementations. The impact of social media, user-generated content and the semantic web is also examined as a key driver in the development of new, more dynamic functionality that is changing the way end-users experience and interact with enterprise content management systems. The conversation encompasses best practices for metadata development and new techniques for the visualization of metadata in DAM user interfaces and workflows.

Journal of Digital Asset Management (2009) 5, 286-297. doi:10.1057/dam.2009.21

Keywords: metadata; taxonomy; search; schemas; ontology; social media

MM: We're here with Tom Bachmann of EAS Planning. Tom can you give us a little bit of your background?

TB: I'm a principal at EAS Planning; we are a small consultancy founded in 2001. I'm sort of a hybrid between an information science specialist and a strategic planner. So I tend to tackle a range of issues. Both up-front planning as well as more granular work on metadata solutions involving taxonomy and schema development.

My background is in primary research, so when working on DAM projects I often get involved in solution-adoption among users. I have a Masters in Library and Information Science with a concentration in Information Architecture. Earlier in my career, during the dot-boom era, I worked a lot on Information Architecture and user experience for web content.

MM: Excellent. Would you give us an overview of what we intend to cover in today's session?
TB: OK, we have a couple of different areas: one, how can we help out folks who need to understand the buzzwords around metadata, including taxonomy and schemas and thesauri. Let's say I'm tapped by my organization to develop a DAM solution for the enterprise. What do I need to know about metadata? So we'll unpack and explain some of the basics about metadata structures and terminology. And then, we'll do some more exploratory thinking in part 2 about how metadata is changing and new drivers of change such as the semantic web and social media. Finally, if we have time, we'll get into some interesting examples of metadata visualization: that is, how metadata is displayed in user interfaces for content management and DAM systems.

MM: That sounds like a great way of providing a magisterial summary for the field.

TB: I've recently surveyed several clients about their experiences with implementing DAM 
systems. And if you ask them 'what do you know now that you wish you knew before?' quite a few will answer, 'I wish we had paid more attention to the metadata.' As you know, you can digitize your analog content and you can load that into a content management system, but simply collecting content into one place doesn't make it into assets. The value of assets begins with the ability to locate and re-use, to exchange assets and to leverage that content for myriad purposes. And metadata is key to accomplishing that. So, strong metadata solutions correlate to a more successful and relevant DAM for an organization and from a user perspective.

MM: You could say then that metadata is what makes a file an asset. In particular, it allows you to find the asset, to secure it, to associate with it certain permissions and rights and so on. Metadata allows you to move assets in a controlled manner.

TB: Those are all examples of metadata functioning as part of a business strategy. In order for the metadata to function in that manner, it first has to be properly structured. How are the assets categorized? With what characteristics are they tagged for retrieval? This is an intersection between library science and larger business needs and goals.

It starts with properly structured metadata, and clients often ask, 'What do you mean by metadata?' The conventional definition is 'It's the data about the data.' (Or, in this case, the data that describes one's assets.)

But, for me, thinking about metadata in the mindset of contemporary users, I define it as 'What you know about an asset - it can be the smallest fragment of information - that enables you to find out everything else about that asset.' Think about Googling a fragment of text or a phrase from Shakespeare. From that one phrase, you find out everything else through the relationships in the metadata. The name of the sonnet or play that is the source. The full text of the work. The actors who have famously played leading roles in it. The location of current productions. Video clips of performances. That experience of tapping into that wealth of information is the role that metadata can play for the end user.

MM: This brings up a key point in terms of how many users first come to engage and understand metadata, and it's from the Google experience - the Google search bar. Where I throw a key word and unlock a whole universe of related content.

TB: Right. That Google experience, for better or worse, then forms the expectations of how a DAM system should perform.

MM: In addition to that, people also have a search experience vis-à-vis their corporate intranet or their website, which oftentimes tends to be a really poor and inconsistent experience.

So that's another example of users having a well-lit mental map of what they're looking for.

But the interface and the underlying structure of the interface don't correlate onto that mental map of what should be there.

TB: Here is where we get to the issue of 'What's the difference between what Google is doing and what's happening in an enterprise CMS system or in the search tools on website?' One difference is, Google is using their vast amount of metadata they've gathered to maximize the recall of content related to your query and they are not worrying so much about precision. In fact, they flaunt it. That's why we see billions of hits proudly listed at the top of a result set for a search in Google. But how does that help me as a user? I can only read through how many? Most people won't read beyond the first 10 or 20 listings.

Whereas enterprise CMS systems are often engineered to do the reverse: they're created by librarians who are focused on the precision of the search results. So it's a vastly different experience for the user. In particular, new CMS users are often surprised or frustrated at getting 'no matches found to your search.' 'How can that be?' they think, 'It's supposed to be in there.' And they begin to doubt the integrity or ability of the CMS to work for them. This is a slippery slope as users can be skeptical to start with, and it doesn't take much to discourage them further. If we build our metadata structures with this in mind, we can somewhat compensate for these user expectations that are created by the Google experience.

MM: What can translate into metadata criteria stems from how we organize our personal computers. Oftentimes, the way you organize your stuff will look completely foreign to anyone but you. People tend to have their idiosyncratic world views represented in the folder structure of their file management system. 
TB: Absolutely. And that directory tree of folders, as you say, that also forms expectations of what's going to happen with a DAM. This time, driven by the PC operating system and its 'folder' paradigm. Typically, a DAM is an object-oriented database/search system, so there is no directory tree of folders. There's a search interface instead. In a sense, it is closer to Google. It's never going to behave like the filing system on your PC.

MM: To summarize, the Google experience focuses on reach and comprehensiveness.

Then you have the corporate intranet search experience, which tends to focus on precision and matters specific to the operation of a company. Another aspect of what makes that particular system work is that a lot of people inside the firewall already have a pretty good idea of what they're looking for. As a function of that, they are willing to tolerate a lot more unproductive searches because they haven't quite yet gotten the right search term or argument to return what they wanted. Is that a fair summarization?

TB: Yes. You're right on track. So, once we recognize these factors, a best practice is to take this into account and mitigate against it when designing our search experience or when configuring our metadata and implementing DAM solutions.

MM: And then the third domain of experience and expectation really derives from one's personal experience with personal computing. Simply how you organize your desktop and your folder structure and understanding that the folder structure itself is another form of metadata. A structured hierarchical way of navigating vast fields of files.

TB: This brings to mind innovators that bridge the gap across these different paradigms. From a diverse range of innovators, we are seeing more user interfaces that are developed to combine some of these attributes. They introduce a whole new level of what's expected by the user.

So, for example, Exalead is a small application that I have on my desktop that provides a search interface for everything on my PC. Including faceted search through all of my e-mails and the files in my directory trees. Or you may have interfaces that combine different search experiences - combining the directory tree with the power of Google search. For example, what Getty Images does on their site, and in their search interface combines these different search modes.

As a cycle, we see innovations from the world of Web 2.0 that eventually become a widely held expectation among mainstream users. Taken together, these innovations become the new expected standard. For example, when you put a query in a search interface and it comes back to you with 'Did you mean ...' and it gives you spelling alternatives or alternative search terms. Or you go to Amazon and search for something and it says, 'People who searched for this also browsed for that and the other thing.' These aspects of the search experience rely on metadata structures. In fact, they are derived from a search thesaurus to be more specific.

And as these aspects of the search experience become more widely implemented and 'standard' features, then DAM and CMS vendors will find users expecting this kind of metadata-driven performance for enterprise systems. In effect, they will have to keep up.

MM: So, why do we need metadata?

TB: In the big picture, we need metadata because it transforms our undifferentiated mass of 'content' into findable and re-usable 'assets.' It elevates content to the point where it's being re-used; it's being exchanged. As such, it's a key part of our business strategy.

At a more granular level, what's the metadata doing that's enabling this? It's first allowing us to locate the asset, distinguish the asset from others like it, and retrieve that asset for re-use or sharing. And, in many current implementations, allowing us to automate the movement or delivery of the content out to other systems like a web content management system. So the asset is pushed out to other contexts where it is most relevant and most needed by the enterprise or by its wider network of customers, partners, etc.

Sticking with a user search scenario, the first function of metadata is to respond to that user's search query. To provide the interpretive layer between the language or terms in which the user expresses a query and the contents or assets in the repository. A good example of query terms that need to be interpreted in the DAM context would be 'photos, or pictures, or images, or photographs' - all of 
these synonymous terms have to be disambiguated in some way. If not, I search on the term 'image' and, if the asset is tagged or cataloged as a 'photograph' then I will get back the dreaded 'No results found for your search.'

For a repository that primarily houses 'non-text' assets - photos, video, layouts and illustrations - this is not going to fly. Because rich media assets are not 'text searchable,' the DAM search engine has to rely on a match between the query terms and the tags. So the role of the metadata structures, let's say a controlled vocabulary for tagging assets, is going to be critical to efficiently retrieving assets.

MM: So disambiguate would mean in this case to make it clear and make it unique?

TB: Yes, it's the interpretation of the user's query, the matching of the user's terms with what's indexed in the database and with the terms used in tagging the assets.

Let's say I tag assets with the term 'photographs'; you use the term 'pictures'; and someone else uses 'images.' We all want to get the full set of results back when searching for an image. The controlled vocabulary enables me to retrieve the full set in my keyword search despite the variations in terminology.

That's one scenario involving synonym terms. Other examples include spelling variations, abbreviations or persons with multiple names. We have Elizabeth Taylor, who's also Elizabeth Burton, who's also Elizabeth Fortensky. Or we have the Arab world, which could be the Islamic world, which could also be the Muslim world, which could be the Middle East depending upon the lens you are viewing it through. Those terms may not really be synonyms if we're using them correctly, but they may quite well become interchangeable in their colloquial use among a population of users. In a DAM system, we're dealing with a mass of people who are tagging assets on the fly and another crowd of searchers who are hitting the DAM with queries. And this population often includes a range of designers, video producers or other creative types as well as business people and senior managers who are all searching the DAM. We're going to get all sorts of variations in the query terms. So one responsibility of the metadata structures is to deal with all this variation in the language, to disambiguate it and return the desired results.

MM: One thing that you've done is to introduce the notion of search scenarios. A search scenario is really the confluence of a user's point of view or role within the organization.

A procurement manager has a different point of view than a creative professional. That's part of the search scenario - how do these particular points of view, or user roles, how do they organize this vast field of information?

Part of the search scenario entails not just a use case, but a use case within a workflow, and that workflow is within a larger business process or organization. So search scenarios, anthropologically speaking, are deep and multidimensional.

TB: Yes. And different aspects of those scenarios will play out in what we expect out of the DAM. How will we able to integrate the DAM in user workflows? Which in turn depends on the robustness of the underlying metadata. Are we feeding it with a robust taxonomy? Are we using a search thesaurus? Do we have the fields properly architected in our schema? DAM teams often have high expectations for advance asset management workflows for rich media, but we've got to have the underlying metadata in place to make it possible. Thus, the role of the taxonomy or thesaurus. In fact, these are both flavors of controlled vocabularies. Taxonomy, in particular, is a widely used term that means different things in different contexts.

MM: I think taxonomy is widely used and widely misused.

TB: Exactly. People seem to use it for whatever they want to describe - depending on the context.

MM: Whenever they want to describe something that brings order to a complex mess. TB: Looking closer at taxonomy, we said before that metadata attempts to describe our assets. Now, there are different flavors, or classes, of metadata used in taxonomies. For search and retrieval, we have descriptive, administrative and technical. So, when I have a photograph, if I'm describing what it's about the 'aboutness' or primary subject of it - that is descriptive metadata. It describes who's in it, or where was the image taken. Administrative metadata would be something like the project number for that asset, the file number itself, or the client that it was produced for. Or what 
media outlet was it distributed to. Then, technical metadata would include things like resolution, size, color and other graphic information. Those are the core classes of metadata, and there are other, extended classes.

For example, we may have metadata related to commercial rights, related to security and permission to access, related to long-term preservation and related to enacting more efficient workflows. Those can all be tagged to an asset. The actual terms used in the tagging may be limited to controlled vocabularies that reflect the taxonomy or classification system that we have in place. And the specific facets that we tag on based on the schema we build. So, the schema can be defined as a set of attributes about an asset.

MM: Now, broadly defined, a schema could be as simple as a spider web of linked terms, or it can be something more structured.

TB: To come up with the elements in a schema, we lean on the following question: "What is it that we need to know about an asset in order to achieve our desired workflow?' The elements of the schema are the attributes that inform us about that asset: who created it, for what purpose, what is it about, in what format, etc. Together these schema elements will provide a user with all the critical information about an asset.

In addition, schemas include the rules or syntax for how we express the values in each element. For example, if one element in our schema is 'geographic area' - where a photo was taken - are we going to use a controlled list of countries or regional names or a set of abbreviations for states and provinces or Global Positioning System (GPS) data?

Let's say we have a designer who's creating a layout that's going to go into a publication, and they're grabbing images, and they're putting them into their InDesign file. We'll need to define the key pieces of information about these images as they move through that workflow. First, the designers need to be able to retrieve the image through search. Then, they drop the image into their InDesign layout and eventually save that new layout back into the repository - which includes tagging that new asset using the schema elements or fields that will, in turn, inform the next user in the chain about the current state of the layout.

MM: So, let's talk about some of the industrystandard schemas - Dublin Core represents one starter-kit schema. It comes from OCLC - Online Computer Library Center?

TB: Yes, Dublin Core originated from an OCLC conference in Dublin, Ohio. OCLC is a kind of governing and standards body for large libraries in the US. Anyway, it was developed as a means of describing web-based information and content. Dublin Core has since been incorporated by the W3C, and has a fairly complex life of its own at this point. It includes a basic set of elements like 'Creator', 'Date' and 'Subject.' Beyond that, it now has extended sets of more granular fields for different areas like publishing and rights management.

MM: The W3C is essentially the committee that manages the worldwide web or manages some of the technical definitions for the web.

TB: Yes. Those folks who keep the web organized for all of us.

MM: Then we have this thing called CDWA that was promulgated by the Getty Foundation. Describe that.

TB: Another standardized metadata schema, CDWA is for describing art and architecture objects. It's used primarily in the museum community and archiving communities for those types of objects. So, the schema elements or fields here are specific to scenarios where a curator is working with an art object. For example, what are the pieces of information that a curator needs to know about an object in order to catalog and house and share that art with other institutions? And perhaps re-use it in a variety of installations over time?

MM: So this allows a curator of one cultural heritage organization, be it a gallery library or museum, to describe artifacts in a collection - a physical collection or digital collection. It allows that curator to define a cultural artifact in a standard uniform way that allows other curators at other institutions to use common terms to describe something that's in the original curator's collection.

TB: You've hit on something important about these standardized schemas. They are designed for work processes where you don't have a monolithic, internal, enterprise-centered information domain. But instead, you have a 
federated ecosystem where partners and external users are collaborating with you and you need to be able to search across multiple affiliated repositories of content. Think of all the museums in the Smithsonian or all the libraries in the University of California system that seek to offer federated search. So in order for that to work, you've got to have a standardized set of elements or fields of information that describe each object.

MODS is a schema developed by the Library of Congress. Its roots are in MARC 21, which is a very complex, older system developed for cataloging physical library collections. As Dublin Core began to take hold and become more widely adopted, MODS was created as an alternative. It has some continuity with MARC21 structures, but is much less complex. For organizations seeking to enable federated search or other forms of interoperability, 'crosswalks' can be developed between these standardized schemas. You can imagine that they all share common elements, such as creator, date, a descriptive note and a record ID - crosswalks are a means of establishing equivalency among common fields in these standardized schemas.

MM: Where would you like to go next? TB: Thinking about best practices for the development of DAM systems, once you've done some use cases and scenario work to identify the key fields for your schema, a next step can be to document those in more detail using a data dictionary - this is a another tool to describe, well, what is each field about in the schema. What exactly are we going to put in that field? Why do we have that field? How is the information within the field formatted? What are the values that are acceptable within that field? Let's say we have a rights holder field; we'll want that field or set of fields to communicate the identity of the rights holder and what is their contact information. We'll want to know if there is a legal agreement that is associated with this asset that spells out the rights. If so, we'll need a contract number field for that legal agreement. All of that is documented through a data dictionary. MM: There are specialized software packages just for data modeling and, more specifically, keeping track of data dictionaries. Is that correct?
TB: There are. Quite often they are part of thesaurus-maintenance software. Some DAM systems include modules for this. If you've got an archivist or a library specialist working in a corporate environment, they may have a preferred tool for this. There are a number of firms that offer thesaurus-maintenance software. MM: Who are some leading firms in that field? TB: Well, there are quite a few that range in complexity and features. And more recently, we're seeing more open source tools and freeware. Data Harmony is one provider that I've had good experiences with. Synaptica, which used to be Taxonomy Warehouse, is another well-known firm. WebChoir. MultiTes is widely used. For larger enterprises, firms like SchemaLogic and Autonomy are active in this space.

MM: It makes sense that this data dictionary would make a natural fit as a Software as a Service (SaaS) someplace in the cloud where it simply lives in some well-managed, wellmaintained service that's just brought in as needed.

TB: Yes, maintaining this in the cloud is a viable approach. In terms of metadata maintenance, I think about how do we get away from the robed monks - the acolytes who are in the background orchestrating and controlling all the terms in the metadata and how do we move towards more of a 'folksonomy' solution. That is, how do we help the metadata authorities to incorporate some of the wisdom of the masses and harness things like social tagging in DAM systems? And folksonomy as an approach is gaining ground. Organizations like the $\mathrm{BBC}$ are moving toward collecting the results of social tagging into a folksonomy and then promoting that into their formal taxonomy.

The interesting thing about taxonomies is that we as people are classification machines. Our brains do this automatically all day long. And we're all really good at it. Let's say we're talking with a friend about the Olympics; our brains instantly break down all the Olympic sports - you have the winter sports, the summer sports, the team sports, the individual sports, the water sports and the racquet sports. And our brain is constantly doing this. You walk into a supermarket and why does it intuitively make sense that the produce is to one side and the 
boxed goods are here in the middle and the meats and dairy are over there? We have an innate ability to classify. The success of Delicious and Digg and other social tagging sites is evidence of how well we all do this.

Retailers use this type of innate classification a lot. Particularly online retailers. If I'm selling camping gear, let's say I'm REI; I can construct my site to align with a user's sense of how the domain of camping gear breaks down in subcategories. 'Is this gear for back packing?' 'Is it for rock climbing?' 'Is it for paddling?' 'For cooking?' 'For families camping out with kids?'

MM: In one sense, taxonomy, in this context, is the mind of the market.

TB: Not only that, but on the web it's the means of navigation or the means to findability. It's one pillar in the ability of a website or a DAM system to support interaction between producers and consumers.

MM: Another way of saying that is this particular example of the taxonomy for REI. This represents a habit of mine: We've got a large number of people out there who habitually think in terms of these categories and subcategories. And this works really well for REI and most of the people visiting REI because they already have a pretty welldeveloped mental map of these categories, subcategories and specifically how it corresponds to their lifestyle and activities of the lifestyle.

What I find interesting is that in new markets you don't have these habits of mind established with well-defined shared terms that everyone agrees on. These kinds of taxonomies can be counterproductive; they can be confusing.

TB: That represents a real challenge for emerging markets.

MM: So taxonomies are both the strength and weakness based on how well formed and how well established the market category is.

TB: That role of the authoritative taxonomy developer who is working behind the scenes ... MM: Well, in many respects they are a curator of that particular market.

TB: Right, and if the market is fully formed, the productivity of their work is much higher. In a newer market, you may have to look at a folksonomy model and rely on the productivity of all user-generated knowledge as a way to carve out those emerging, and shared, classifications. There've been some interesting studies around the utility and the effectiveness of user-generated metadata versus authoritative metadata.

MM: What about library sciences?

TB: From the viewpoint of library sciences as a discipline, taxonomy is no longer that buzzword that people wave around to describe anything that they're structuring. It's a more precise form - one type of a controlled vocabulary. Controlled vocabularies increase in complexity as you move from basic synonym rings to authority files to taxonomies to thesauri. The thesaurus is the Cadillac of controlled vocabularies. When we look at user experience for CMS systems, including DAMs, a well-developed thesaurus will enable your users to do the most, have the greatest range of options and functionality at their fingertips.

MM: Would it be fair to say that if you were a fly on the wall, and you were to make an audio recording of all the conversations that your marketing and creative people had, transcribed it and then text-mined it looking for specific key words and phrases, that would begin to identify some of the underlying concepts of your thesaurus.

TB: Yes, that would be one technique in thesaurus and metadata development. So we do things like a content audit and in-person ethnographic work with the creative teams to document and list-out their day-to-day terminology and capture those in our controlled vocabulary lists.

MM: Ethnographic analysis derives from anthropology and was made popular by many of the pioneering anthropologists like Margaret Meade or Levi Strauss. Or they would simply take detailed notes of what a particular native did. So those would be all the things that an ethnographic study would catalog as well as then enumerate as far as how many times did a person or animal do those things by context. Is that a fair characterization of ethnographic research?

TB: It is. And in the context of content management, what we're doing is adapting that technique and focusing it around discovery of key terms and a more in-depth understanding of user language, user workflows and user needs. MM: So, if you said that the thesaurus is the crown jewel of a CMS and/or a DAM, it then follows that the specific elements of that 
thesaurus constitute a class of digital assets. About how long does it take a mid- to a large-sized firm to actually develop and codify its thesaurus say for a marketing operation? TB: My experience has been that most organizations have some of this in place already when we come in. So we're adapting and re-using these existing assets. It's not a terrifically long process, but it's fairly labor-intensive. Often this is a parallel track to the other activities that get your DAM up and running: planning for application development, configuration, planning for storage, planning for networking. One of those tracks is going to be 'OK, let's do the work to define the elements in the schema and to articulate these controlled vocabularies and structured sets of related terms in the taxonomy.' Now, if you articulate and codify a controlled vocabulary for a marketing operation, what you have is a snapshot in time. Think of it as a still frame in a video. Because the next day there's a new media form, there's a new client, there's a new project. And all those terms are forever proliferating.

MM: For many companies, especially in regulated industries, where they have a well-developed corporate intranet, many of the terms associated with the domains and collections and ways of talking about stuff have already been instantiated or expressed in the structure of that intranet. Is that a fair characterization?

TB: Yes. So what we're looking at is adapting it to the needs of the CMS. One of those needs can be 'What's the long-term path for this as a building block system in collaboration with other parts of the enterprise - a records and archiving system or a web content management system?' From a technology strategy standpoint, how are these systems going to work together over time?

OK, now we've unpacked and dispensed with several of these buzzwords on our list thesauri and schemas and controlled vocabularies we're left with ontologies and also topic maps.

MM: So would you say topic maps and ontologies are synonymous?

TB: They're separate things. The way I look at it, these two structures are more often applied in work that goes beyond the core needs of a DAM system. But I think they're still relevant, particularly when we think about what's going to happen next in content management technology. I think that what's happening with both semantic functionality and with social media is going to provide opportunities for DAM systems to do more, to give users a better experience and to become more valuable to their organizations.

MM: So then ontologies are the user's experience of using metadata and taxonomy and keywords?

TB: Ontologies are the a priori classification systems that we all carry around with us in our heads - that represent our understanding of the world. They're the reason why a classification feels right or doesn't feel right when we encounter a large repository for the first time whether it's a supermarket, a giant big-box Lowe's or a complex website. I look at a website like Epicurious and I see how all those recipes are broken down by time of day breakfast lunch and dinner - by regional cuisines like French or Italian and by main ingredients and it all makes great sense to me ... when we bump into these categorical distinctions, why do they make sense to us intuitively? Or why do they look confusing to us? It depends on their degree of alignment with the ontologies that we bring to the party.

Among the computer science community, ontologies are often used in the development of semantic functionality. For example, tools for distilling human interactions into machineinterpretable information rely on ontologies. We'll come back to that.

Topic maps are similar. Let's say you have a taxonomy. OK, it's really just several lists of terms that are related to each other in a hierarchy. A topic map will take those terms, and will start visually expressing relationships between them. Often it does this in the context of a workflow or a task. For example, let's say I'm developing a calendar application for Facebook. And not only is it going to allow me to share calendars with friends, but it's also going to allow me to book appointments and make meeting times and places with others. Depending on how much automation we're going to build into this, we'll need metadata structures to describe the relationship between these parties and break down the appointment-booking process into discrete tasks. Then we'll need to represent those tasks in machine-interpretable information, 
like XML mark-up. So, let's say you're the optician and I'm the client. And I'm going to set up an appointment with you through my web agent. The topic map articulates the connection between us and provides a kind of holding structure for all the pieces of information generated by the task of booking the appointment.

MM: A topic map is used then by a designer of a user experience, an interface and an application such as a calendaring system?

TB: That's right. Topic maps, like taxonomies, come in many forms. So a simple topic map can be a kind of 'mindmap' visual picture of how subject terms relate to each other. And they get more involved from there.

MM: It's also conceivable that the topic map, whether it's as simple as a mind map or something more structured, could in fact become a navigational device in and of itself, correct? You can embed a hyperlink right down to the pixel.

TB: Yes, at a certain point with semantic computing, what you're doing is surfacing the values held in each of the elements of the topic map into the actual interface and therefore into the experience for the user. And similarly, as this relates to DAM implementations, people are looking at the taxonomy and saying let's take this whole set of hierarchical keywords and push them right into the User Interface (UI) so they will display to users and be available to users for things like faceted search.

MM: So one limited but meaningful expression of that would be a tag cloud that you see at someone's blog. And search within a search; when you search within a search, you get a smaller tag cloud.

TB: Good example. The visual of the tag cloud is built off the schema fields and the tags from all those fields. These can be displayed visually as a cloud or they can be presented as a panel on the side of the interface. So we can break down a large collection of photographs by facets like location, number of people, gender of the people, age of the people, emotional subject matter of the photos, genre of the photos, landscapes versus portraits, etc.

MM: This also then gets into the desktop idiom that we described earlier: You click on one of the folders and all the nested folders within the folder show up in the next column.
Then you click on one of those folders and the adjacent column lights up with all the elements of that folder.

So you're able to move right across the desktop, from one column to the next. I'm drilling down, down, down to finer and finer grained items of the nested folders.

Is that a fair characterization?

TB: Yes. And what we're seeing now with search UIs is the blend of these different search modes. So, you may have the folder interface happening with faceted search options next to that in a side panel; then next to that you have a visual mind map which may display as a sort of spider-web of subject topics. Check out Kartoo.com or Ujiko.com or the AquaBrowser for examples of these.

MM: Another dimension that I want to bring forward in this notion of multimodal search is that, for the most part, the person searching in basic system terms constitutes either a named or an unnamed user. But there's no inherent metadata associated with the searcher. There's no subscription profile where I specify I have particular interest in certain categories of information, media formats, disciplines or topics.

As I navigate these search results, as I start to interact in this multimodal search mode, my preference, or to take another biological term, my preferenda now provides a new way of suppressing less relevant stuff and surfacing more relevant stuff given my engagement preferenda.

TB: That's right out of the social media playbook. As we can bring that together with what's happening with multimodal searching, we get closer to intelligent interfaces and the semantic web.

MM: And a third dimension to bring into that would then be engaging content so as to induce a transaction.

So now you could imagine at an Amazon. com-type of website, there's all this content that I'm navigating with multimodal search. I've got engagement preferenda - who I am and what I'm interested in. That together then creates a personal tag cloud. So as I'm looking at any one particular result, I've got a tag cloud assembled as a function of this synchronization or inferences between my engagement preferenda and what's actually there in the site as it's related to that search result. Then the system can infer from that what banner ads or sponsor 
content would be most relevant to someone with that preferenda.

TB: As you go through that scenario, the site or the application running on the site can be pulling together our user behavior and our preferences and capturing those into an ontology that reflects the perspective or mindset of the user. Their own personal preferences and ontology created on the fly! The question is can we automate that and make it machine-interpretable and coordinate it with other metadata structures. It remains to be seen.

MM: So this topic of ontology becomes much richer because now we can bring forward the notion of semantic tagging, correct?

TB: Let's look more closely at the term 'semantic.' This expresses the notion that humans recognize the significance of a piece of information according to a specific context or task. For example, I'm trying to book a trip to Australia, and I want to get a good deal on my flight. When I see a bunch of travel information on Expedia, I judge it through that lens.

In other words, the value of each piece of information about each flight is based on my quest of getting a good deal on my flight. And on how I define what is a 'good deal.'

Similarly, the idea behind semantic technology is to enable machine-interpretable descriptions of information and services. So, now the application can derive the same contextual meaning from that information on Expedia that I would. In essence, let's get the application to work as an agent that sees the contextual meaning of the information much as we would.

Then the application is better able to help us with completing the task. The application knows I'm trying to go to Australia. It knows that I want to book a cheap flight, but that I also want to fly direct not through Shanghai. It knows that I need an appointment with the dermatologist and that I prefer a dermatologist who is covered by my insurance. And within easy driving distance and one that has availability in the not too distant future. So, the application recognizes the significance of the information involved in the task. The metadata structures we've been talking about are one of the keys to making this work.

MM: Let's introduce some of those emerging standards for organizing content and assets vis-à-vis the expectations and the habits of mind that have emerged due to social media.

TB: OK, if you think about the social media landscape, it is a prolific source of new content let's say we look at it as 'one massive, federated repository of user-generated content.' Content provided by Facebook and Wordpress and Blogger and Twitter and Flickr and whoever else. How are these social media tools helping their members and users breakdown and deal with all of this content? In effect, can they make it all more accessible, findable, shareable and re-usable? Well, this is what DAM systems are designed to accomplish for organizations dealing with large collections of digital content. So, I think there's little doubt that, over time, these realms will cross-pollinate and that better user experiences for content management will emerge from this.

Already there is overlap. Take a look at sites that are specifically designed to search usergenerated content - sites like Omgili.com and Whonu.org - and they're all about breaking this content down and making it accessible and usable. I think content management systems could really benefit from the tools that are being innovated in social networking sites - the next generation of Facebook competitors. As well as social tagging sites and content sharing sites like Flickr.

So the success of social media is really good news for organizations seeking to manage large collections of content. A lot of organizations go through multiple attempts at making content management work within their enterprise before they get it right. So why does that happen? One hopes that from these failures, the applications will get a little bit smarter and the organizations will be a little more focused about what they need.

MM: And user expectations became crisper. TB: Right, and user expectations and behavior patterns became crisper based on the experiences they have with Web 2.0 technologies. And so a lot of organizations are looking at how to make CMS and DAM really work at a higher level in the next iteration.

MM: It seems to me that most content management systems have a fundamental challenge to not breach in their content-governance model. Specifically, most enterprise content management systems have an allergic reaction to 
user-generated content. There's no discussion or interaction. There might be a corporate blog, but it tends to be a highly constrained interaction.

Social media and the larger social networking that generates the media breaks the contentgovernance model of most firms. And as a function of that, most of these firms with broken content-governance models are essentially not going to be able to effectively harness the upside as well as the downside dynamics of social media and the larger social networking. This at the end of the day becomes digital word of mouth, online advocacy, everything else that stimulates awareness, drives consideration and ultimately induces a transaction.

TB: Many organizations are intent on reaching this next generation of consumers or influencers or decision-makers. And I think we see more clearly now that the old governance model will be a non-starter for these targets. And that social media is how a whole generation experiences the world. This is where the eyeballs of those consumers have migrated. And the fingertips. They're authoring their own wealth of new content at a tremendous rate.

MM: And they want to mash up what you have online with the conversations they have with their friends. Currently, it's difficult for your content to become currency in those mashed-up conversations among friends.

TB: I agree. We have to look more closely at how we enable participation in those conversations and whether our content management systems can interact with these wider social networks. Do we have the right tools and widgets and applications that can play in the environments where our audience spends all their time, such as on Facebook and its competitors?

MM: So this gets into another interesting point that came out of a conversation I had with Trae Clevenger of Targetbase, who is an expert in multichannel analytics. He says that the data are conclusive and definitive that most online consumers visit no more than 12 locations on a regular basis. For many of us, our daily habit would be four or five places. There seems to be a natural instinct; you could almost call it a biological or neurological constraint.

People don't visit more than 12 places on a regular basis. One of those places tends to be
Facebook. While there are 200 million people on Facebook today, in three years there will be a billion people on Facebook.

TB: Right. There will also be fragmentation. MM: There will be massive fragmentation. Currently, out of the 200 million of the Facebook users today, about 50 to 60 million of those users go to Facebook 50 times a day. So Facebook is no longer a social-networking platform. It is quite literally a social, cultural interface to my network.

TB: It's like their portal.

MM: It's even beyond a portal. It's become their interface. A portal is something that you go to. An interface is something that you come from. It's an engagement interface.

Trae believes that you have to create multimodal content to syndicate right into Facebook.

People first engage with you there and then go to a lifestyle destination microsite, which is tightly linked with Facebook plus other social networks like MiVo or Twitter or whatever, and then back to the product mothership or product site. A lot of the socialization happens at Facebook and these specialized microsites.

If we're going to wait for the Fords and the Krafts and FedEx's to revamp their CMS systems so they're more like WordPress in terms of being social, then they'll miss 10 years of market: Can't get there from here.

TB: Interesting. In the social media experience, these user activities travel across instances and locations. And as that is occurring, I keep thinking that there are these sorts of 'repository moments' that happen from time to time. This is what grabs my attention because it's those repository moments that require the kind of metadata structures we've been discussing. And they are vital opportunities for companies seeking to connect and do business with people.

MM: This gets to the root cause as far as why most content management systems have a broken governance model. The inherent assumption of content governance is what I'll call a locked room so that when you put content into the CMS you're basically putting it into a locked controlled environment.

And a DAM is, if you will, a safety deposit box within the vault. You need to have a very specific key to get into the locked content behind the firewall of the larger CMS or 
corporation's intranet. All of this governance becomes a brick shithouse into which you now find yourself locked.

The new social governance model basically says, 'Whatever conversation, whatever piece of media, whatever link my customers use to engage me I have to now encapsulate. I have to be able to capture, to have a meta-tag asset within a larger content-governance protocol. But I have to clearly mark out that this piece of content actually 'lives' at Amazon or Facebook. Not only do I have to capture the asset, but also I then have to capture it as an asset metadata and all the policies associated with it, describing business policies as it relates to what I can do with this. But I also have to maintain a link back to the actual instance of that in its native environment or in its generative environment, that being Facebook or LinkedIn.' TB: Yes. That also gets to auto-categorization, auto-classification that is used to deal with the volume of that social media output - to categorize and classify it.

MM: Now what you're talking about is what's referred to as social media monitoring and specific kinds of spidering capabilities that crawl through forums and discussions, classifying content as it relates to your market, and then further summarizes those in terms of positive, neutral and negative sentiment, and then summarizes that in terms of the overall temperature of the brand in very specific consumption cohorts.

TB: Now you're seeing some overlap of that and search communities. Do you know Omgili. com? The name stands for 'Oh My God I Love It.' It's a search engine designed to spider, to crawl all of the UGC content on the web and make it searchable.

MM: Fabulous.

TB: They're taking the established search engine algorithms, and applying them to social media communications. Crawling them all, indexing them and making them searchable with a web interface. For example, you want to find out more about Michael Moon to bring him in to speak at your forum; you can see what's happening on all the chatrooms about him. Another one is whonu.com. These search interfaces are built for breaking down social content and allowing you to filter it. Another example of the kind of cross-pollination that will dramatically affect the development of content management and DAM systems in the near future. It's coming and the sooner we recognize that it's coming, the better able we'll be to plan for it and take advantage of it.

MM: Excellent. Let's stop here. Thank you, Tom. 